\title{
Quantifying Information Leaks using Reliability Analysis
}

\author{
Quoc-Sang Phan* Pasquale Malacaria* Corina S. Păsăreanu ${ }^{\dagger}$ Marcelo d'Amorim \\ * Queen Mary University of \\ London, UK \\ \{q.phan,p.malacaria\}@qmul.ac.uk \\ $\dagger$ Carnegie Mellon Silicon Valley, \\ NASA Ames, USA \\ corina.s.pasareanu@nasa.gov \\ ¥ Federal University of \\ Pernambuco, Brazil \\ damorim@cin.ufpe.br
}

\begin{abstract}
We report on our work-in-progress into the use of reliability analysis to quantify information leaks. In recent work we have proposed a software reliability analysis technique that uses symbolic execution and model counting to quantify the probability of reaching designated program states, e.g. assert violations, under uncertainty conditions in the environment. The technique has many applications beyond reliability analysis, ranging from program understanding and debugging to analysis of cyber-physical systems. In this paper we report on a novel application of the technique, namely Quantitative Information Flow analysis (QIF). The goal of QIF is to measure information leakage of a program by using information-theoretic metrics such as Shannon entropy or Rényi entropy. We exploit the model counting engine of the reliability analyzer over symbolic program paths, to compute an upper bound of the maximum leakage over all possible distributions of the confidential data.

We have implemented our approach into a prototype tool, called QILURA, and explore its effectiveness on a number of case studies.
\end{abstract}

\section{Categories and Subject Descriptors}

D.2.4 [Software/Program Verification]: Formal methods; D.4.6 [Security and Protection]: Information flow controls

\section{General Terms}

Algorithms, Performance, Security, Theory, Verification

\section{Keywords}

Quantitative Information Flow; Reliability Analysis; Model Counting; Symbolic Execution

\section{INTRODUCTION}

Quantitative information flow analysis (QIF [7, 14]) is a rigorous approach to measuring information leakage. The intuition is that absolute security is hard to achieve, consequently, under some circumstances, programs with small

Permission to make digital or hard copies of all or part of this work for personal or classroom use is granted without fee provided that copies are not made or distributed for profit or commercial advantage and that copies bear this notice and the full citation on the first page. Copyrights for components of this work owned by others than ACM must be honored. Abstracting with credit is permitted. To copy otherwise, or republish, to post on servers or to redistribute to lists, requires prior specific permission and/or a fee. Request permissions from Permissions@acm.org.

SPIN'14, July 21 - 22 2014, San Jose, CA, USA

Copyright 2014 ACM 978-1-4503-2452-6/14/07 ...\$15.00.

http://dx.doi.org/10.1145/2632362.2632367
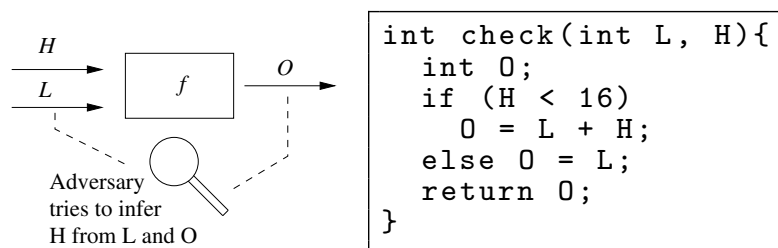

Figure 1: $2^{4}$ (=4 bits) distinct outputs for $H>0$.

leaks are acceptable as secure. QIF has gained considerable attention in recent years. It has been used to analyze software confidentiality $[4,10,16]$, to measure loss of anonymity in communication protocols [6, 19], and to assess leakage of information via side-channels [8]. QIF builds on the hypothesis that a malicious user can make observations on the public input and output data used in a function call to infer confidential data. The technique measures the reduction in uncertainty about the secret as an hypothetical malicious user makes observations on public data.

In recent work we have developed a software reliability analysis technique [9] that uses a bounded symbolic execution to collect a set of symbolic paths over the analyzed programs. The path constraints associated with the paths are combined with given probabilistic usage profiles and analyzed using model counting techniques [1] to quantify the probability of reaching designated program states (e.g. successful termination or the opposite, failure states such as assert violations). In this work we adapt the reliability analysis to QIF by considering information leakage as the failure states and using model counting over the input constraints to quantify the likelihood of leakage assuming a uniform usage profile.

Example. Figure 1 shows an example function that we use to illustrate QIF. It is a convention in the security literature to use the label $L$ ("low") to denote non-sensitive input, to use the label $H$ ("high") to denote sensitive private input, and to use the label $O$ ("output") to denote the output. A malicious user has access to the public data, $L$ and $O$, and tries to infer the hidden secret, $H$, from that.

Automating QIF analysis is a challenge. For example, to analyze the program above, in [16] and more recently [17], the authors manually transformed it into bit vector predicates. Other papers require users to have verification expertise to use an interactive theorem prover [12], or require user to write a driver following a template [10] or to instrument the program under test [13].

In this paper, we introduce an automated tool, QILURA (Quantify Information Leaks Using Reliability Analysis), for QIF analysis. Given a program, and inputs labeled as high and low, QILURA computes an upper bound on the 
maximum number of bits that the program can leak to a public observer. Our implementation is done in the context of Java bytecode programs and the SPF [21] symbolic execution engine, extended for reliability analysis [9]. However, the work is general and can be applied in the context of any programming language for which a symbolic execution tool exists.

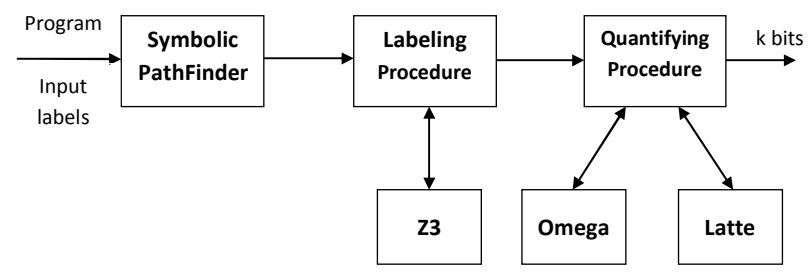

Figure 2: Architecture of QILURA

At a high level, the architecture of QILURA is depicted in Figure 2. The user labels the inputs of the program with high and low. The program is then passed to SPF to collect all possible symbolic paths. The Labeling Procedure, using a finegrained self-composition [5], classifies all the paths into three categories: clean, direct and indirect. The procedure uses z3 [3] for satisfiability checking of self-composition condition.

Finally, the Quantifying Procedure uses model-counting techniques [1] over the symbolic constraints (simplified using Omega [2]) collected by SPF to count the number of inputs that follow paths labeled with "direct" and provides an upper bound of $k$ bit on the leaks.

\section{PRELIMINARIES}

\subsection{Quantitative Information Flow}

Consider again the program in Figure 1 in the case $L=$ $0 \times 1000$. Clearly, only integer values from $0 \times 1000$ to $0 \times 100 f$ are possible outputs for this function. An attacker has hence 16 possible output choices depending on the value of $H$ : observing outputs $0 \times 1001 . .0 \times 100 f$ reveals that $H$ is in the range $[1,15]$ and observing output $0 \times 1000$ reveals that the secret is 0 or greater than 15 .

Let $X_{H}, X_{L}$ and $X_{O}$ be random variables representing the distribution of $H, L$, and and $O$, respectively. Assuming the attacker only knows that $H$ is a 32 -bit variable, his $a$ priori probability of guessing the value of $H$ in one try is $\frac{1}{2^{32}}$. which leads to the uncertainty of $H$ measured in Rényi's min-entropy [22] is: $E\left(X_{H}\right)=-\log _{2}\left(\frac{1}{2^{32}}\right)$. Moreover, the expected probability of guessing the secret in one try after observing the outputs is:

$$
\frac{15}{2^{32}}+\frac{2^{32}-15}{2^{32}} \frac{1}{2^{32}-15}=\frac{15}{2^{32}}+\frac{1}{2^{32}}=\frac{16}{2^{32}}
$$

which leads to the remaining uncertainty after observing $O$ is: $E\left(X_{H} \mid X_{L}=0 \times 1000, X_{O}\right)=-\log _{2}\left(\frac{16}{2^{32}}\right)$. Leakage is calculated as the reduction of uncertainty of $\mathrm{H}$ after the observation

$$
\Delta_{E}\left(X_{H}\right)=E\left(X_{H}\right)-E\left(X_{H} \mid X_{L}=0 \times 1000, X_{O}\right)=\log _{2}(16)
$$

Notice that $\log (16)=\log$ (number of output observations): this is not a coincidence. A fundamental QIF result (the channel capacity theorem [15, 22]) shows that leakage for a program is always less than or equal to the log of the number of observables of the program. More importantly the result holds if we consider not only the above notion of leakage based on the probability of guessing the secret [22] but also the notion of leakage based on Shannon's information theory measuring the number of bits leaked [7]. For these reasons counting the number of observables is the basis of state-ofthe-art QIF analysis, e.g. [16, 10, 20, 13, 17, 19], and also the basis for this work. The channel capacity theorem also justifies the following:

DeFInITION 1. Given a program $P, Q I F$ is the problem of counting $N$, the number of possible outputs of $P . \log _{2}(N)$ is the channel capacity (i.e. the maximum leakage) of the program $P$, denoted by $C C(P)$, measured by Shannon entropy or Renyi's min-entropy.

\subsection{Symbolic Execution}

Symbolic Execution (SE) [11], is a program analysis technique which executes programs on unspecified inputs, by using symbolic inputs instead of concrete data. For each executed program path, the analysis builds a path condition $p c$, i.e. a conjunction of boolean conditions characterizing the inputs that follow that path. This $p c$ is built according to the branching conditions in the code and it is checked for satisfiability using off-the-shelf solvers. If a $p c$ becomes unsatisfiable it means that the corresponding path is not feasible in the program (and the analysis backtracks). The execution paths followed during the symbolic execution of a program are characterized by a symbolic execution tree. The nodes represent program (symbolic) states and the arcs represent transitions between states.

In the setting of SE, the program $P$ is modelled as a (treelike) transition system: $P=(\Sigma, I, F, T)$, where $\Sigma$ is the set of symbolic states; $I \subseteq \Sigma$ is the set of initial symbolic states; $F \subseteq \Sigma$ is the set of final symbolic states; and $T \subseteq \Sigma \times \Sigma$ is the transition function. A symbolic path of $P$ is represented by a sequence of symbolic states: $\rho=\sigma_{0} \sigma_{1} . . \sigma_{n}$, such that $\sigma_{0} \in I, \sigma_{n} \in F$ and $\left\langle\sigma_{i}, \sigma_{i+1}\right\rangle \in T$ for all $i \in\{0, \ldots, n-1\}$. The symbolic semantics of $P$ is then defined as the set of all symbolic paths $\mathcal{R}$ (i.e. the symbolic execution tree). We define two functions init and fin to get the initial state and final state of $\rho$ : $\operatorname{init}(\rho)=\sigma_{0}$ and $\operatorname{fin}(\rho)=\sigma_{n}$.

We denote by $\left.X\right|_{y}$ the value of the variable $X$ at the state $y$. After symbolically executing the program $P$ with initial input symbols $H=\alpha, L=\beta$, for each $\sigma_{i} \in F$, i.e. each leaf of the symbolic execution tree, we have a symbolic formula $f_{i}$ for the value of the output $O$ in the symbolic environment: $\left.O\right|_{\sigma_{i}}=f_{i}(\alpha, \beta)$. The path condition $p c_{i}$ is a formula $c_{i}(\alpha, \beta)$ expressing the condition for state $\sigma_{i}$ to be reachable. Each $p c_{i}$ corresponds to a symbolic path $\rho_{i}$. We define the function path such that: $\operatorname{path}\left(\rho_{i}\right)=p c_{i} \equiv c_{i}(\alpha, \beta)$.

\subsection{Reliability Analysis}

In previous work we described a reliability analysis tool [9] based on symbolic execution and model counting. The tool takes as input a Java program and the usage profile and computes an estimate of the probability for satisfying (or violating) a property of interest, e.g. an assertion in the code or a designated observable event. Internally, the tool uses a bounded symbolic execution (SPF [21]) to produce a set of path constraints which are then classified in the sets $p c^{T}$ and $p c^{F}$ based on whether the paths lead to the the target event $(\mathrm{T})$ or not $(\mathrm{F})$. A third set $p c^{G}$ (G stands for "grey") characterizes the paths for which the event did not occur, but the bound was hit (e.g. due to loops or 
recursion). The path constraints define disjoint input sets and cover the whole input domain of the program [11]. The tool then quantifies the probability of each set. Note that the computed probability for $p c^{G}$ results in a measure for the confidence in the results obtained within the bound (the lower the probability the higher the confidence); see [9]. For the sake of space we will not consider grey paths here; we will instead label them as " $T$ ".

The probability of satisfying the property is defined as the probability of an input distributed according to the usage profile to satisfy any of the path constraints in $p c^{T}$. Assuming a uniform usage profile, this probability is \# $\left(p c^{T}\right) / \#(D)$, where $\#\left(p c^{T}\right)$ is the number of solutions satisfying the disjoint constraints in $p c^{T}$ and $\#(D)$ is the size of the finite (but possibly very large) input domain $D$. \# $\left(p c^{T}\right)$ can be computed efficiently using model-counting techniques such as Latte [1]. For QILURA we do not compute probabilities but use directly the counts over the computed symbolic constraints.

\section{QILURA}

At a high level, QILURA performs a two-step analysis. First, SE is run to collect all symbolic paths of the program (up to a user-specified depth), then each path is assigned a label: (i) clean: if it leaks no information, (ii) direct: if it leaks information via direct flow, and (iii) indirect: if it leaks information via indirect flow. Secondly, a model counter for symbolic paths from [9] is used to count the number of possible inputs that go to "direct" paths, and compute an upper bound on the leakage. QILURA is available at: https://github.com/qif/jpf-qilura.

\subsection{Fine-grained self-composition}

Given a program $P$ that takes secret input $\mathrm{H}$, public input $L$ and producing public output $O$, we denote by $P^{\prime}$ the same program as $P$, with all variables renamed: $H$ as $H^{\prime}, L$ as $L^{\prime}$ and $O$ as $O^{\prime}$. Following [5] we express self-composition by the Hoare triple:

$$
\left\{L=L^{\prime}\right\} P ; P^{\prime}\left\{O=O^{\prime}\right\}
$$

This Hoare triple states that if the precondition $L=L^{\prime}$ holds, then after the execution of $P ; P^{\prime}$, the postcondition $O=O^{\prime}$ also holds. Thus, satisfying the triple guarantees that the program $P$ does not leak information.

We run $\mathrm{SE}$ on the self-composed program $P ; P^{\prime}$ with input symbols as follows: $H=\alpha, H^{\prime}=\alpha_{1}, L=L^{\prime}=\beta$. Thus the precondition automatically holds. Assume the symbolic semantics of $P$ and $P^{\prime}$ is $\mathcal{R}$ and $\mathcal{R}^{\prime}$ respectively. The selfcomposition formula in (1) can be re-written as:

$$
\forall \rho \in \mathcal{R},\left.\rho^{\prime} \in \mathcal{R}^{\prime} \cdot \operatorname{path}(\rho) \wedge \operatorname{path}\left(\rho^{\prime}\right) \rightarrow O\right|_{f i n(\rho)}=\left.O^{\prime}\right|_{f i n\left(\rho^{\prime}\right)}
$$

In case (2) is violated, if $\rho^{\prime}$ and $\rho$ are the same symbolic path up to renaming then $\rho$ leaks information via direct flow, otherwise $\rho$ and $\rho^{\prime}$ leak information via indirect flow.

The implementation for checking self-composition is built from (2) as in Figure 3. The function isSAT is implemented by calling the SMT solver z3 [3].

\subsection{Model counting for symbolic paths}

For a symbolic path $\rho$, let \#in $(\rho)$ and \#out $(\rho)$ denote the number of concrete inputs and outputs of $\rho$ respectively. Obviously $\# i n\left(\rho_{i}\right)$ is $\#\left(p c_{i}\right)$ computed in [9]. After being

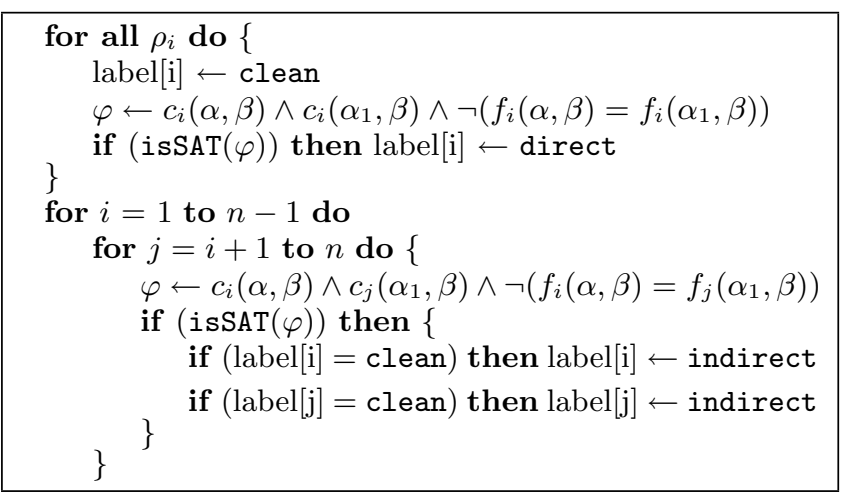

Figure 3: Fine-grained self-composition

labeled, all paths are classified into three categories: clean, direct and indirect. So the channel capacity is bounded by:

$$
C C(P) \leq \log _{2}\left(\Sigma \# \text { out }\left(\rho_{c}\right)+\Sigma \# \text { out }\left(\rho_{i}\right)+\Sigma \# \text { out }\left(\rho_{d}\right)\right)
$$

where $\rho_{c}$ is the clean path, $\rho_{i}$ is the indirect path, and $\rho_{d}$ is the indirect path.

- Since clean paths are not interfered by the confidential input we can replace $\Sigma \#$ out $\left(\rho_{c}\right)$ with 1 .

- An indirect path only reveals that the program follows that path, its output is not interfered, and each path has one output. Thus, $\Sigma \#$ out $\left(\rho_{i}\right)$ is just the number of indirect paths.

- We hence only need to compute $\Sigma \#$ out $\left(\rho_{d}\right)$.

A deterministic program can be viewed as a function that maps each input to exactly one output (denotational semantics). Therefore, the number of inputs is always greater than or equal to the number of possible outputs. This means $\#$ in $(\rho) \geq \#$ out $(\rho)$, and $\Sigma \#$ in $\left(\rho_{d}\right) \geq \Sigma \#$ out $\left(\rho_{d}\right)$.

By using the model counting engine for symbolic paths in [9], we can compute $\Sigma \# i n\left(\rho_{d}\right)$, and hence compute an upper bound of channel capacity $C C(P)$.

\section{EVALUATION}

Automated QIF analysis is notoriously hard. To the best of our knowledge, the only tool for QIF analysis of Java bytecode is our own work jpf-qif [20] which uses SE for QIF analysis, but no model counting. Instead jpf-qif adds the conditions for testing each bit of the output at the end of the program, hence exploring all these conditions using SPF. We compare jpf-qif with QILURA below.

We also compare with BitPattern [16], which computes an upper bound on channel capacity by exploring the relations between every pair of bits of the output. In more recent work [17], BitPattern was improved using new heuristics. We compare QILURA with (the improved) BitPattern on several case studies taken from $[16,17]$.

Moreover, we consider a special case when the program does not leak any information to assess the effectiveness and precision of our technique in

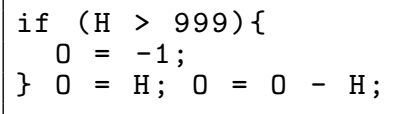

Case Study 1: No Flow such a corner case. The program does not leak information because the output $O$ is always 0 regardless of the value of the secret $H$. However, the assignment $O=H$ and the 


\begin{tabular}{|l|c|c|c|c|c|c|}
\hline \multirow{2}{*}{ Case Study } & \multicolumn{2}{|c|}{ jpf-qif } & \multicolumn{2}{c|}{ QILURA } & \multicolumn{2}{c|}{ BitPattern } \\
\cline { 2 - 7 } & Capacity & Time & Upper Bound & Time & Upper Bound & Time \\
\hline No Flow & 0 & 2.304 & 0 & 0.790 & - & - \\
\hline Sanity check, base =0x00001000 & 4 & 45.324 & 4.09 & 1.066 & 4 & 0.036 \\
\hline Sanity check, base =0x7ffffffa & 4 & 35.346 & 4.09 & 1.049 & 4.59 & 0.203 \\
\hline Implicit Flow & 2.81 & 0.897 & 3 & 0.796 & 3 & 0.011 \\
\hline Electronic Purse & 2 & 1.169 & 2.32 & 0.854 & 2 & 0.157 \\
\hline Ten random outputs & 3.32 & 1.050 & 3.32 & 0.814 & 18.645 & 0.224 \\
\hline
\end{tabular}

Figure 4: Capacity and bounds are in bits, times are in seconds. "_" means "not reported".

condition $H>999$ make the program be rejected by other qualitative information-flow techniques, e.g. the ones based on type systems or taint analysis.

\section{Results and discussions}

Figure 4 summaries our experiment, we take the time from the faster version of BitPattern in [17]. Note that in both [16] and [17], the authors manually transform the programs into bit vector predicates, so there will be extra time if they automate this process.

As shown in the figure, the upper bounds computed by QILURA only deviate to a small extent from the exact channel capacities computed by jpf-qif. However, by using a model counting tool, QILURA is much faster.

The BitPattern technique can also compute rather tight upper bounds in most of the cases. However, by analysing the relations of pairs of bits, the technique is vulnerable when possible values of the output are not in a specific range, as shown in the last case study.

\section{RELATED WORK}

Backes et al. [4] describe how to use the model checker ARMC and Latte for QIF analysis. Their technique is very precise but also extremely expensive: it involves input counting to compute the pre-image of the observables; in contrast our input counting is used for counting the observable. The work was extended in [12] which uses $\mathrm{KeY}$, an interactive theorem prover, instead of ARMC, but requires significant user effort.

The only technique that can precisely determine if a program leaks information is self-composition [5]. QILURA also uses self-composition with the key difference that it is able to determine if a single symbolic path leaks information. A preliminary idea on verifying self-composition using SE has been reported in a student workshop [18].

\section{CONCLUSION AND FUTURE WORK}

In this paper we presented QILURA which embodies a novel application of reliability analysis based on symbolic execution and model counting to Quantitative Information Flow analysis. QILURA is still just a prototype but our preliminary experiments show encouraging results. We plan to perform larger case studies, and to investigate approximate exploration techniques (instead of the exact, complete exploration presented here), for increased scalability, but with formal statistical guarantees on the results.

\section{ACKNOWLEDGMENT.}

We wish to thank Antonio Filieri for his support during the development of QILURA. This work is partially supported by the Google Summer of Code 2013 program.

\section{REFERENCES}

[1] Latte. http://www.math.ucdavis.edu/ latte/.

[2] Omega. http://www.cs.umd.edu/projects/omega/.

[3] Z3. http://z3.codeplex.com/.

[4] Backes, M., Kopf, B., And Rybalchenko, A. Automatic discovery and quantification of information leaks. SP '09, IEEE Computer Society, pp. 141-153.

[5] Barthe, G., D'Argenio, P. R., And Rezk, T. Secure information flow by self-composition. CSFW '04.

[6] Biondi, F., Legay, A., Traonouez, L.-M., And WAsowski, A. Quail: A quantitative security analyzer for imperative code. CAV'13, Springer-Verlag.

[7] Clark, D., Hunt, S., and Malacaria, P. A static analysis for quantifying information flow in a simple imperative language. J. Comput. Secur. 15, 3.

[8] Doychev, G., Feld, D., Köpf, B., Mauborgne, L., And Reineke, J. Cacheaudit: A tool for the static analysis of cache side channels. SEC'13, pp. 431-446.

[9] Filieri, A., Păsăreanu, C. S., and Visser, W. Reliability analysis in symbolic pathfinder. ICSE '13.

[10] Heusser, J., ANd Malacaria, P. Quantifying information leaks in software. ACSAC '10, ACM.

[11] King, J. C. Symbolic execution and program testing. Commun. ACM 19, 7 (July 1976), 385-394.

[12] Klebanov, V. Precise quantitative information flow analysis using symbolic model counting. QASA '12.

[13] Klebanov, V., Manthey, N., and Muise, C. Sat-based analysis and quantification of information flow in programs. vol. 8054 of QEST '13. pp. 177-192.

[14] Malacaria, P. Assessing security threats of looping constructs. POPL '07, ACM, pp. 225-235.

[15] Malacaria, P., And Chen, H. Lagrange multipliers and maximum information leakage in different observational models. PLAS '08, ACM, pp. 135-146.

[16] Meng, Z., And Smith, G. Calculating bounds on information leakage using two-bit patterns. PLAS '11.

[17] Meng, Z., And Smith, G. Faster two-bit pattern analysis of leakage. QASA '13.

[18] Phan, Q.-S. Self-composition by symbolic execution. ICCSW' '14, pp. 95-102.

[19] Phan, Q.-S., AND Malacaria, P. Abstract model counting: a novel approach for quantification of information leaks. ASIACCS '14, ACM.

[20] Phan, Q.-S., Malacaria, P., Tkachuk, O., AND PĂSĂREANU, C. S. Symbolic quantitative information flow. SIGSOFT Softw. Eng. Notes 37, 6, 1-5.

[21] Păsăreanu, C. S., And Rungta, N. Symbolic pathfinder: symbolic execution of java bytecode. ASE '10, ACM.

[22] Sмiтh, G. On the foundations of quantitative information flow. FOSSACS '09, pp. 288-302. 